\title{
CRITERIOS A CONSIDERAR PARA LA FINANCIACIÓN DE LA EDUCACIÓN SUPERIOR EN VENEZUELA
}

\author{
CRITERIA TO BE CONSIDERED FOR FUNDING OF \\ HIGHER EDUCATION IN VENEZUELA
CRITÉRIOS A CONSIDERAR PARA O FINANCIAMENTO DA EDUCAÇÃO SUPERIOR NA VENEZUELA

Luis E. Torres-Nuñez ${ }^{1}$

\begin{abstract}
RESUMEN
La creciente incapacidad económica que presentan los Estados de la región latinoamericana para financiar las universidades públicas, ha posicionado el financiamiento público como un tema común en casi todas las reformas dirigidas a la educación superior. La insuficiencia de recursos públicos está comprometiendo la efectividad de la gestión académica en las universidades públicas, sin que aún se conozcan los asuntos económicos claves para el fortalecimiento de las políticas públicas del sector. Este ensayo tiene como propósito: proponer un nuevo sistema de criterios para la financiación de la educación superior; partiendo del análisis de la experiencia venezolana en materia de las asignaciones de los recursos públicos empleados para el financiamiento de las instituciones de educación superior durante el periodo 2000-2015. Es una investigación de carácter documental. La propuesta contiene siete (7) elementos integradores que darían lugar al establecimiento de una política de financiamiento para la educación superior. Finalmente, se sugiere que el Estado supere los viejos esquemas de presupuesto negociado "incremental", las fórmulas de financiación "rígidas" y las asignaciones diferidas, que no logran identificar las necesidades y costes básicos para ser asociados a la gestión académica de las instituciones de educación superior, en función de promover la eficiencia, equidad y descentralización en el financiamiento público destinado al sistema de educación superior venezolano.
\end{abstract}

PALABRAS CLAVE: Política de financiamiento. Educación superior. Presupuesto universitario. Gestión financiera.

\begin{abstract}
The growing economic inability in Latin-American states to finance public universities, has positioned public funding as a common theme in almost all targeted reforms of higher education. Insufficient public resources are compromising the effectiveness of academic management in public universities, without knowing the key economic issues for strengthening public sector policies. This essay is intended to: propose a new system of criteria for funding of higher education; based on the analysis of the Venezuelan experience in allocations of public resources used to finance higher education institutions during the 2000-2015 period. It is a documentary research. The proposal contains seven (7) integrating elements that would lead to the establishment of a funding policy for higher education. Finally, it is suggested that the State exceeds the old negotiated "incremental "schemes, "rigid" budget formulas and deferred funding allocations, which fail to identify basic needs and costs associated to the academic management of higher education institutions, in terms of promoting efficiency, equity and decentralization in public funding for the Venezuelan higher education system.
\end{abstract}

KEYWORDS: Financing policy. Higher education. University budget. Financial management..

\section{RESUMEN}

A crescente incapacidade econômica que apresentam os Estados da região latino-americana para financiar as universidades públicas posicionou o financiamento público como um tema comum em quase todas as reformas

\footnotetext{
${ }^{1}$ Estudiante del Doctorado Latinoamericano en Educación: políticas y profesión docente. Sede UPELVenezuela. Universidad Nacional Experimental Sur del Lago. E-mail: torresl@unesur.edu.ve - ORCID: http://orcid.org/0000-0002-2005-9101
}

Submetido em: 14/10/2016 - Aceito em: 12/11/2016.

\begin{tabular}{l|l|l|l|l|l|l} 
(C) Rev. Inter. Educ. Sup. & Campinas, SP & v.2 & n.3 & p.450-460 & set./dez. 2016 & ISSN 2446-9424
\end{tabular}


dirigidas à educação superior. A insuficiência de recursos públicos está comprometendo a efetividade da gestão acadêmica nas universidades públicas, sim que ainda se conheçam os assuntos econômicos-clave para o fortalecimento das políticas públicas do setor. Este ensaio tem como propósito propor um novo sistema de critérios para o financiamento da educação superior; partindo da análise da experiência venezuelana em matéria das designações dos recursos públicos empregados para o financiamento das instituições de educação superior durante o período 2000-2015. É uma investigação de caráter documental. A proposta contém sete (7) elementos integradores que dariam lugar ao estabelecimento de uma política de financiamento para a educação superior. Finalmente, se sugere que o Estado supere os velhos esquemas de orçamento negociado "incremental", as fórmulas de financiamento "rígidas" e as designações diferidas, que não conseguem identificar as necessidades e custos básicos para ser associados à gestão acadêmica das instituições de educação superior, em função de promover a eficiência, equidade e descentralização no financiamento público destinado ao sistema de educação superior venezuelano.

PAlaVRAS-CHAVE: Política de financiamento. Educação superior. Orçamento universitário. Gestão financeira.

\section{Introducción}

El financiamiento público de la educación superior (ES) en Latinoamérica continúa siendo la fuente principal de los ingresos de las instituciones de educación superior (IES); razón por la cual, ha sido uno de los aspectos más debatidos en las tres últimas décadas, principalmente por la influencia e impacto que genera la desproporción entre los crecientes índices alcanzados por la masificación estudiantil con respecto a los "casi" estables índices de la inversión para los recursos públicos empleados para dicho sector. Sin lugar a dudas, esta situación ha conllevado a los Estados latinoamericanos a considerar el tema del financiamiento público como un elemento común en casi todas las reformas dirigidas a la ES, sin que aún se conozcan respuestas de asuntos claves para el fortalecimiento de las políticas públicas del sector: ¿cuánto cuesta la ES y cuánto debería costar? ¿cuánto se gasta efectivamente en la ES y cuánto se invierte en la ES? ¿quiénes soportan el costo de la ES y quiénes se benefician?, en síntesis, a pesar que el financiamiento ha sido un tema ampliamente discutido en las últimas décadas, ello no ha significado mayor conocimiento en materia del financiamiento (GARCÍA-GUADILLA, 2004).

Generalmente es argumentado por los investigadores y los hacedores de políticas, que los recursos públicos son -y posiblemente siempre sean- insuficientes para el adecuado desempeño de la gestión académica en las universidades latinoamericanas; en tal sentido el presente estudio, tiene como propósito presentar una propuesta de criterios a ser considerados para la financiación de la educación superior en Venezuela, prestando particular atención a los posibles mecanismos implementados para la asignación de recursos públicos para las IES, toda vez que se procura promover los principios de equidad y descentralización en la distribución de los fondos públicos.

Este ensayo se organiza en dos dimensiones a saber: Caracterización del financiamiento de la educación superior en Venezuela 2000-2015 y líneas generales para establecer una política de financiamiento universitario en Venezuela. 


\section{Caracterización del financiamiento de la educación superior en Venezuela 2000-2015}

El financiamiento de la educación superior en Venezuela se ha caracterizado por ser de tipo "negociado"2 y destinado históricamente a la oferta ${ }^{3}$. En función de facilitar la comprensión del fenómeno de estudio, es preciso señalar que el financiamiento de la ES para el caso venezolano, ha sufrido modificaciones sustantivas en los últimos quince años, los cuales podrían señalarse por separado en tres sub-periodos a saber: 2000-2005; 2005-2010 y; 2010-2014. Los cuales serán fundamentados por los trabajos de García-Guadilla (2006) y Parra-Sandoval (2011).

Para el primer sub-periodo 2000-2005, se observa la existencia de un modelo de asignación de recursos presupuestarios de tipo mixto, es decir, en primer lugar, prevalece el modelo "histórico negociado" (al igual que en toda la región latinoamericana), solo que se procuró darle mayor relevancia a algunos indicadores de referencia, tales como: 1) número de estudiantes y egresados; 2) actividad de investigación; 3) proyectos especiales y actividades de postgrados; 4) número de jubilados y 5) relación empleado-profesor.

En segundo lugar, la aplicación de un modelo basado en ciertos indicadores conocidos como "Normas CNU-1992", , contemplaban que al menos el 18,50\% de la proporción del presupuesto universitario debería responder a los criterios obligatorios (fijos y variables) como mecanismo de financiamiento condicionado para la ES; estos criterios obligatorios se discriminaron en dos grupos: 1) los coeficientes fijos, los cuales alcanzan una proporción del 13,50\% distribuidos de la siguiente forma: a) coeficientes de investigación fijo (3\%); b) coeficiente para postgrado $(1,5 \%)$; c) coeficiente para bibliotecas $(1,5 \%)$; d) coeficientes para la extensión y cultura $(1,5 \%)$; e) coeficiente para deportes $(1,5 \%)$; f) coeficientes para funcionamiento y equipamiento de pregrado $(1,5 \%) ; \mathrm{g}$ ) coeficiente para desarrollo de recursos humanos $(0,5 \%) ; \mathrm{h})$ coeficientes para convenios institucionales $(0,5 \%)$; i) coeficiente para crecimiento y desarrollo $(0,5 \%) ; \mathrm{j})$ coeficiente para mantenimiento $(0,75) ; \mathrm{k})$ seguro de asistencia médica para los estudiantes (0,25\%). 2) los coeficientes variables, que solo están establecidos para: coeficiente de investigación variable (5\%).

Este mecanismo de financiamiento, podríamos atribuirle como fortaleza, la intencionalidad de precisar que al menos un $18,50 \%$ de los recursos asignados para las IES contribuyeran al fortalecimiento de la misión universitaria, lejos del gasto de personal y de consumo corriente no vinculado a la mencionada misión; como debilidad, señalamos que los

\footnotetext{
${ }^{2}$ El presupuesto negociado es un mecanismo que se caracteriza por establecer acuerdos entre el Estado y las IES para las asignaciones de recursos financieros, los cuales generalmente obedecen a los criterios de la negociación con tendencia histórica incremental; la distribución de los recursos puede ser establecida en los presupuestos por conceptos (como ocurrió en el caso venezolano con la aplicación de las normas CNU, hasta antes el año 2005) y/o presupuesto en bloques (como ha estado ocurriendo en el caso venezolano posterior al año 2005, asignaciones globales de la cuota presupuestaria sin ninguna otra consideración).

${ }^{3}$ Se conocen como fondos destinados a la oferta, cuando los mismos son asignados a las IES por el Estado, por dos motivaciones concretas: a) para financiar los costes de la enseñanza y b) para financiar los costes de la investigación (GARCÍA DE FANELLY, 2007).

${ }^{4}$ Este modelo de financiamiento venezolano fue uno de los pioneros en Latinoamérica en la década de los años 90 del siglo anterior.
} 
indicadores eran aplicados a todas las IES en "igualdad"5 de condiciones, sin tomar en consideración las particularidades y diferencias existentes de tipo geográficas, tecnológicas y de desarrollo científico en las IES, situación que procuró siempre mayores recursos económicos para las universidades históricamente consolidadas, complicando el desarrollo científico integral de todo el SES, principalmente de las universidades experimentales y las otras IES.

A pesar la modalidad de condicionamiento del financiamiento ya descritas para los años 2000-2005 (establecimiento del presupuesto condicionado por una proporción del 18,5\% según las normas CNU-1992), lamentablemente los presupuestos universitarios no dejaron de presenciar históricamente el mismo comportamiento del gasto público: $95 \%$ para el gasto corriente y a veces un poco menos del $5 \%$ para la inversión y desarrollo. Lo que se constituye como un incumplimiento "flagrante" de los criterios establecidos en las normas CNU-1992. Situación que conllevó al gobierno a implementar algunos mecanismos de recursos adicionales, mediante la implementación de un proyecto que tendría como propósito "la excelencia académica universitaria con equidad, eficiencia y mayor pertinencia social", proyecto que después culminó siendo el proyecto "Alma Mater" (FUENMAYOR TORO, 2004).

Para el sub-periodo 2005-2010 se observa el rompimiento del mecanismo mixto de financiamiento aplicado desde la década anterior (1995-2005), por un nuevo mecanismo de centralización y diferimiento de los fondos públicos asignados a las IES. En este sub-periodo, al menos dos situaciones condicionaron la asignación de recursos económicos; nos referimos en primer lugar, a la modificación de la técnica presupuestaria para todos los órganos de la administración del Estado y los entes descentralizados, en este último caso, las universidades. Según el artículo 2 del Reglamento número 1 de la Ley Orgánica de Administración Financiera para el Sector Público (RBV, 2005). La modificación de la estructura presupuestaria-financiera de las universidades, ocasiono que las universidades públicas dejar atrás el viejo esquema de formulación presupuestaria situado en los 10 programas históricos, para pasar al establecimiento de los nuevos mecanismos "proyectos y acciones centralizadas". Ello rompió en alguna medida con la trayectoria del modelo anterior del cómo y para qué se distribuían y asignaban los recursos a las IES. En segundo lugar, el cambio de la mayoría del equipo técnico del Ministerio de Educación Superior -como se llamaba para el año 2005- agudizó en lo sucesivo el problema de la falta de soporte "técnico", ya que el nuevo equipo no encontró mayor viabilidad para reformular y replantear un nuevo modelo de asignación de recursos financieros para las universidades.

Es así como a partir del 2006 el Ministerio implementa la asignación de recursos públicos sin que se conozcan los mecanismos y criterios aplicados; este nuevo modo de asignar recursos se caracteriza en primer lugar, por direccionar en bloque, recursos discrecionales por parte de los órganos del Estado para las IES; y en segundo lugar, por la implementación como nuevo elemento de financiación de la solicitud de recursos adicionales por parte de las IES, como consecuencia de la existencia de insuficiencias presupuestarias, para lo cual es necesario la tramitación de créditos adicionales (mayormente para gastos de funcionamiento, salarios y providencias estudiantiles). Los cuales a su vez son aprobados por la Asamblea Nacional, han sido administrados como mecanismos de fondos centralizados por

\footnotetext{
${ }^{5}$ Se precisa, que en este caso la igualdad promovió la inequidad financiera que imposibilita la igualdad de oportunidades para el acceso de los recursos públicos de las IES.
}

\begin{tabular}{l|l|l|l|l|l|l} 
(C) Rev. Inter. Educ. Sup. & Campinas, SP & v.2 & n.3 & p.450-460 & set./dez. 2016 & ISSN 2446-9424
\end{tabular}


el Estado ${ }^{6}$; sin que en los dos casos exista referencia alguna sobre cuáles son los criterios transparentes y públicos- empleados por el órgano central del Estado para la distribución de los recursos públicos a las IES.

En este mismo sub-periodo (2005-2010), se registra un aumento significativo de la matrícula universitaria como resultado de la política de municipalización de la ES, llevada a cabo principalmente por la "Misión Sucre". Sin embargo, la falta de financiamiento suficiente y oportuno para la implementación de dicha estrategia de municipalización condujo a las IES a una profunda crisis presupuestaria y salarial por la que han tenido que transitar hasta el momento en que se escribe el presente ensayo, toda vez que las IES han tenido que compartir sus recursos financieros con la Misión Sucre para la contratación del nuevo personal y otros gastos logísticos necesarios para la atención de al menos 1915 nuevas sedes para la ES, requeridas por la nueva modalidad de ALDEAS universitarias ${ }^{7}$.

El sub-periodo 2010-2015, se ha caracterizado en lo financiero por mantener la continuidad y profundización de la estrategia de asignaciones empleadas en el sub-periodo anterior, la cual consiste en primer lugar, las asignaciones en bloques discrecionales de los recursos ordinarios dirigidos a las IES; y en segundo lugar, la solicitud de recursos adicionales (insuficiencias presupuestarias) por parte de las IES, para la tramitación de créditos adicionales para gastos de funcionamiento, salarios y providencias estudiantiles, conceptos diferentes a la inversión tecnológica y científica que requieren las IES. Razón suficiente para comprender que se continúa con la inexistencia de una política de financiamiento de recursos públicos que procuren la asignación de estos recursos con criterios de equidad y justa distribución para todas las IES en el país.

Este último periodo ha evidenciado un decrecimiento económico (no se alcanzan en valores absolutos las asignaciones presupuestarias de los años anteriores en relación con los ajustes de las tasas inflacionarias) de hasta seis veces en el presupuesto destinado a todas las IES. Así lo señalan los precios constantes en los presupuestos asignados durante los años 2010-2014; además de ello, con los mismos presupuestos "a valores constantes", se han financiado dos contrataciones colectivas para todos los trabajadores (activos y jubilados) del sector universitario, como mecanismo de ajuste extemporáneo del acentuado rezago de los salarios durante varios años en el contexto de una economía inflacionaria. Estas dos situaciones, indican con claridad el por qué las IES están en este momento con escasos recursos disponibles, para financiar la operatividad y ninguna inversión en la gestión académica de las IES en Venezuela.

Finalmente, en este punto, se evidencia, en primer lugar, la falta de una política de financiamiento que se articule con los recursos económicos disponibles y los resultados esperados de las demás políticas públicas dispuestas por el Estado venezolano en materia de educación superior. En segundo lugar, la centralización excesiva de los recursos para el

\footnotetext{
${ }^{6}$ Se considera Fondos Centralizados por el Estado, aquellos recursos económicos solicitados por las IES y aprobados por la Asamblea Nacional, pero que a posterior son administrados discrecionalmente por el órgano central del Estado sin ninguna regulación.

${ }^{7}$ Los Ambientes Locales de Desarrollo Educativo Alternativo Socialista (ALDEAS) universitarias, constituyen una nueva red de espacios (aulas de escuelas, casas de cultura, centros penitenciarios, aulas de IES e instalaciones propias de reciente construcción) para el desarrollo de la Misión Sucre (PARRA-SANDOVAL, 2014).
}

\begin{tabular}{l|l|l|l|l|l|l} 
(C) Rev. Inter. Educ. Sup. & Campinas, SP & v.2 & n.3 & p.450-460 & set./dez. 2016 & ISSN 2446-9424
\end{tabular}


funcionamiento de las IES y las asignaciones discrecionales por parte de los órganos del Estado incrementa la tensión política en las relaciones del Estado con las IES, toda vez que entorpecen el adecuado desenvolvimiento de las operaciones que debe emprender la gestión académica de las instituciones universitarias. En tercer lugar, también es evidente la falta de diversificación de las fuentes de ingresos para las IES, que les permitan ampliar la inversión para el desarrollo de la infraestructura física, científica y tecnológica, requerida para sus fines académicos, además para el establecimiento de fondos salariales concursables adicionales, que contribuyan al fortalecimiento económico de la profesión académica, y frenen la fuga de académicos a otros sectores del mercado laboral nacional e internacional.

\section{Líneas generales para establecer una política de financiamiento universitario en Venezuela}

Antes de plantear las líneas gruesas para establecer una política de financiamiento universitario en Venezuela es importante hacer referencia a algunas consideraciones previas que le den un contexto filosófico y legal sobre las diversas relaciones que deben establecerse entre el Estado y las IES, y para ello discriminamos las siguientes:

En primer lugar, se debe tener claro, que el éxito de cualquier política pública radica en su planificación e implementación, la cual requiere estar respaldada por la participación seria y responsable de todas las partes involucradas para el establecimiento de los acuerdos. En tal sentido, cualquier política pública para la ES (incluida la del financiamiento), tendrá éxito en la medida que todos los órganos del Estado vinculados a la educación superior y los diferentes actores de las IES logren precisar la mayor cantidad de puntos de coincidencia para su formulación y posterior aplicación.

En segundo lugar, el Estado debe construir una postura firme para preservar la protección del carácter "público" de la ES, evitando la aplicación de mecanismos de tipo mercado para procurar ampliar recursos adicionales para el financiamiento de la ES, de acuerdo con los planteamientos realizados por el Acuerdo General sobre Comercio de Servicio (AGCS).

En tercer lugar, definir y reorganizar la plataforma institucional de todo el SES venezolano; iniciar por precisar cuáles son las funciones y alcances de los órganos del Estado $\mathrm{y}$, cuáles son los planteamientos concretos que se espera en el cumplimiento de la misión universitaria en materia de profesionalización, generación de ciencia-tecnología y preservación de la cultura por parte de la IES. En este punto es preciso señalar que existe una correspondencia entre lo que se concibe como la "misión" de las IES o de sus SES y los tipos de financiación que en ella pueden ser aplicados, Venezuela está en presencia de un SES de carácter "público" por ello la misión de las IES deben estar influenciada para procurar la formación de profesionales de alto nivel que procuren la satisfacción de las necesidades colectivas y el desarrollo de su sociedad, para ello el Estado está obligado a implementar mecanismos de financiamiento que procuren la igualdad distributiva de los recursos públicos como la garantía de una gestión universitaria efectiva (SANYAL; MARTIN, 2006). 
En cuarto lugar, se deben impulsar mecanismos diferenciados para el financiamiento de la gestión académica; es decir, establecer categorías para las IES y un grupo de criterios diferenciados para financiar la formación del pregrado, la generación y el desarrollo científico y las actividades de extensión. Este apartado será aplicado en la siguiente propuesta de política de financiamiento, la cual tiene sus fundamentos teóricos según lo expresado por (SANYAL; MARTIN, 2006; SALMI; HAUPTMAN 2006).

En quinto lugar, se debe crear un programa nacional para la atención integral y efectiva de las providencias estudiantiles. Entre otras consideraciones se debe precisar los costos operativos de los servicios de alimentación, transporte y atención medica; además se deberá establecer una plataforma única de registro nacional para conocer los beneficiarios de las providencias estudiantiles; con el firme propósito de priorizar el financiamiento de las becas para aquellos estudiantes provenientes de los estratos sociales IV y V; asimismo, evitar la duplicidad de beneficiarios en diferentes plazas ofertadas por el Estado venezolano o las IES.

En sexto lugar, se debe impulsar una política de gestión electrónica en todo el SES, toda vez que se procura en primer lugar, una disminución considerable de los costos de papelería y demás suministros de imprenta y reproducción. En segundo lugar, una contribución sostenible para el desarrollo económico del país.

Ahora bien, entre las generalidades que podría contener una estructura sistémica para la política de financiamiento, que tome en cuenta lo anterior y permita a las IES, un acceso equitativo en la distribución, ejecución y control de los recursos púbicos por el Estado, podríamos señalar los siguientes siete (7) elementos, lo cual a su vez son una reconsideración de los seis (6) planteamientos propuestos por (Sanyal; Martin, 2006):

a) Diversificación de las fuentes de financiamiento distintas a la fuente pública. En Venezuela existen algunas experiencias que bien vale la pena revisar, redefinir, contextualizar e implementar. Nos referimos a:

- Generación de ingresos captados del sector empresarial, la experiencia inmediata ha sido la aplicación de la Ley Orgánica de Ciencia y Tecnología. Es un mecanismo de inmediata recaudación para el establecimiento y se debe establecer un fondo concursable que le permita a las IES corregir, ajustar y superar la insuficiente financiación pública percibida por el Estado, que ha impedido el desarrollo y fortalecimiento de la gestión académica en su totalidad.

Este enfoque permitirá superar el principal cuestionamiento de toda la experiencia, es que se ha situado en los extremos de su aplicación. En primer lugar, los recursos fueron dispuestos para el concurso de las IES bajo unos criterios absolutos que desfavorecían el concurso equitativo y efectivo de las IES de reciente creación y las de poca capacidad de desarrollo científico (en concreto era un mecanismo excluyente para propiciar el desarrollo científico integral del SES venezolano) y, en segundo lugar, producto de la modificación de la ley, los recursos fueron dispuestos para ser centralizados en su totalidad por parte de los órganos del Estado, en donde se ha dificultado a las IES y la comunidad académica, conocer cuáles son los mecanismos de concursos para las asignaciones de recursos y su correspondencia con los planes previstos.

\begin{tabular}{l|l|l|l|l|l|l} 
(C) Rev. Inter. Educ. Sup. & Campinas, SP & v.2 & n.3 & p.450-460 & set./dez. 2016 & ISSN 2446-9424 \\
\hline
\end{tabular}


- Establecimiento de fundaciones. El establecimiento de fundaciones universitarias ha sido otra experiencia venezolana que procuro en el pasado recursos adicionales, sin lugar a dudas es una recomendación vigente para las IES venezolanas, el establecimiento de este tipo de órganos propios dedicados a la administración de recursos económicos, que se ocasionen de los resultados de la gestión científicatecnológica, asesorías y consultorías al sector público y privado, entre otras actividades productivas propias de los activos que gestionen las IES.

Es necesario precisar la personalidad jurídica de la fundación universitaria, toda vez que debe facilitar la implantación de mecanismos efectivos de transparencia, auditoria, control, supervisión y custodia de los fondos gestionados. Logrando superar los actos de corrupción e ineficiencia que albergaron muchas empresas réntales, por constituirse como figuras jurídicas ajenas a la condición universitaria.

- Gestión de las reservas liquidas por parte de las IES. Hasta hace pocos años, las IES, una vez conocido y honrado el cronograma de desembolso de los recursos para el año por parte del Estado, se dedicaban a gestionar algunos instrumentos financieros en la banca nacional, con el firme propósito de generar recursos adicionales que les permitieran subsanar costos puntuales, que en ocasiones no podían ser satisfechos mediante el presupuesto ordinario.

La aplicación de este apartado requiere de al menos dos consideraciones. En primer lugar, se debe reglamentar cuales son los conceptos de los recursos universitarios que pueden ser sujetos de las gestiones liquidas en la banca comercial, así como su frecuencia y la determinación de su límite máximo de tiempo. En segundo lugar, se debe aplicar en época de una economía estable en términos de inflación.

b) Asignación racional de los recursos ordinarios en bloque para cada IES que les permita la cobertura de los costos fijos "normados por estudiante" 8 para su funcionamiento (gasto de personal, materiales y servicios).

Para este caso, se podrían diseñar varias fórmulas, a saber: en primer lugar, fórmulas de financiación basadas en los insumos básicos requeridos para la gestión universitaria ${ }^{9}$, toda vez que se procura identificar varios elementos base, que permitan establecer una correlación entre las variables: número del personal administrativo y obrero, la relación del personal académico y la matrícula y, la demanda de insumos según las actividades académicasadministrativas previstas; En segundo lugar, fórmulas de financiación basadas en la matrícula y costes por estudiante para establecer las diferencias entre los costos de estudiantes discriminados por las diferentes áreas del conocimiento, puesto que todos no demandan los mismos insumos y en menor sentido sus cantidades; incluso estos costos podrán ser considerados por regiones geográficas. En ambos casos es recomendable establecer las proporciones, toda vez que se procura favorecer la gestión académica de las IES.

c) Establecimiento de un fondo de subvenciones en bloque para la investigación y el establecimiento de otras retribuciones salariales para los investigadores. Se propone en

\footnotetext{
${ }^{8}$ En los costes normativos por estudiantes, se incluyen coeficientes óptimos entre el personal y los estudiantes además de otras medidas que procuran mejorar la eficiencia económica del gasto. (Salmi; Hauptman; 2006).

${ }^{9}$ Es una de las primeras fórmulas diseñadas por los Estados y las IES. (Salmi; Hauptman; 2006).
}

\begin{tabular}{l|l|l|l|l|l|l}
\hline (C) Rev. Inter. Educ. Sup. & Campinas, SP & v.2 & n.3 & p.450-460 & set./dez. 2016 & ISSN 2446-9424
\end{tabular} 
este punto el establecimiento de un Fondo de desarrollo científico "categorizado" ${ }^{10 "}$ con indicadores de desarrollo y progreso científico, de cooperación y asociación entre las IES de diferentes categorías, entre otros criterios transparentes para su concurso.

Nos referimos a la categorización de las IES y la categorización de los científicos del país; en primer lugar, se deben diferenciar las capacidades científicas y tecnológicas que poseen las instituciones y sus académicos, toda vez que se procura un concurso equitativo entre los pares. Para ello se pueden tomar en consideración algunos elementos propios de la actividad investigativa como podrían ser: la evaluación de proyectos de investigación, la capacidad científica demostrada por la IES, creación de centros de investigación de excelente capacidad científica y la determinación de la prioridad científica para los Estados y la sociedad en general.

d) Establecimiento de un fondo de subvenciones para las providencias estudiantiles. Este fondo podrá emplear diferentes modalidades a saber:

- Subvenciones que otorga directamente el Estado y las IES a los estudiantes que ya se encuentran dentro del SES toda vez que se procura la prosecución y culminación efectiva de los estudios de aquellos alumnos que se encuentren en situaciones de vulnerabilidad económica. Estas subvenciones son para contribuir a sufragar los costes de manutención y residencia de los estudiantes con escasos recursos (principalmente estratos sociales IV y V).

- Fórmulas de financiación basadas en insumos para los gastos de operatividad y mantenimiento del servicio de transporte, comedor y atención médica. Generalmente estas fórmulas son diseñadas por los Estados y las IES, para procurar identificar varios elementos base, que permitan establecer una correlación entre las variables: número de unidades de transporte, rutas empleadas y estudiantes beneficiados; número de bandejas a ofertar en el almuerzo y cenas, sus costos en la zona geográfica y su relación con los estudiantes beneficiados; definición de los servicios de atención medica básica y su relación con los insumos requeridos.

e) Producción de indicadores académicos, económicos y de impacto social: para poder establecer cualquier sistema integral de financiamiento público, es indispensable conocer las estadísticas de las IES y de los diferentes órganos del Estado involucrados en la gestión del SES. La producción de indicadores, procura apartarse solo de los índices económicos y resultados académicos que imposibilitan el análisis particular de las IES y a su vez la comprensión del impacto social y científico que está ejerciendo todo el SES. Debe quedar en claro, que la inexistencia de este elemento, dificultará emplear con efectividad cualquier fórmula de control y evaluación sistémica que procure el mecanismo de ajustes requerido por el SES en Venezuela.

f) Utilización de los recursos: esta fase es conocida por muchos técnicos como la debida instrumentación de la vinculación Plan-Presupuesto, y es que precisamente el objetivo de

\footnotetext{
${ }^{10}$ Los fondos categóricos constituyen otra alternativa de asignación de fondos públicos a las IES; a través de este medio el Estado puede asignar recursos con finalidad específica a varias IES y a los científicos, toda vez que procura corregir o ajustar una financiación insuficiente en el pasado, real y percibida. (Salmi; Hauptman; 2006).
}

\begin{tabular}{l|l|l|l|l|l|l} 
(C) Rev. Inter. Educ. Sup. & Campinas, SP & v.2 & n.3 & p.450-460 & set./dez. 2016 & ISSN 2446-9424 \\
\hline
\end{tabular}


este elemento es identificar todas las actividades que demandan la utilización o empleo de cualquier tipo de recursos (humanos, financieros, materiales y técnicos) más allá de las fuentes de las cuales los ha obtenido, de tal manera que facilita a las IES el proceso de rendición de cuentas públicas a lo cual están obligadas y al Estado el control de la gestión universitaria, logística y económica de las IES.

g) Evaluación y auditoria: son estos dos aspectos poco desarrollados en la gestión universitaria que llevan adelante las diferentes IES; siempre se está presentando algún desbalance entre la relación de la gestión académica, sus resultados y la vinculación con los costes y/o recursos consumidos. Como elemento positivo, se empieza a observar a nivel mundial, la tendencia a publicar en diferentes medios de difusión los documentos oficiales sobre la rendición de cuentas de las IES de los recursos entregados por el Estado; sin embargo, es una política que se debe convenir para instrumentar efectivamente los mecanismos de captación y difusión de información pública, que evite en todo caso la omisión, manipulación y errores de los datos publicados.

\section{Consideraciones finales}

El fenómeno de la expansión de la ES continuará su crecimiento acelerado en los países de nuestra región, y sin un debido tratamiento constituirá la profundización de la actual crisis estructural que experimentan los SES presentes en la Latinoamérica, como lo es en este justo momento el caso venezolano. Ahora bien, los autores señalan que, para superar los estados de crisis de la masificación de la ES, las políticas públicas deben apuntar a promover mayores mecanismos expeditos de financiamiento para que las universidades cumplan con eficiencia su compromiso social.

Estos mecanismos expeditos de financiamiento, requieren que los Estados superen los viejos esquemas de presupuesto negociado de tipo "incremental" y las fórmulas de financiación "rígidas", que no favorecen la identificación de las necesidades y coste básicos asociados al soporte económico de la gestión académica de las IES. En tal sentido, el mayor debate acá planteado subyace en la relación del Estado-Universidad, con el propósito de construir una nueva política de financiación pública, partiendo de los elementos técnicos que han resultado con éxito en diversas partes del mundo y que desde luego no son posibles replicarlos sin una adecuada reconsideración crítica y contextualización social, económica, política y académica.

Otro de los aspectos significativos, es que urge diversificar los ingresos que se destinan al mantenimiento del SES venezolano. Se presentaron algunas consideraciones generales que pretenden promover una discusión amplia entre el Estado y las IES, con el objeto de convenir las acciones conjuntas que contribuyan a materializar el compromiso social de las IES. Se trata pues de que las universidades gestionen y accedan a los recursos de la mejor manera, estableciendo mecanismos de rendición de cuentas de sus resultados; sin tomar en consideración si sus recursos son de origen público o privado. 


\section{Referencias}

FUENMAYOR TORO, Luis. El financiamiento universitario. Educere, Año 8, $\mathrm{N}^{\circ}$ 26, p. 385-395. 2004. disponible en:

<http://www.saber.ula.ve/bitstream/123456789/19887/2/articulo12.pdf > consultado el: 09 de abril de 2010.

GARCÍA DE FANELLY, Ana. La reforma universitaria impulsada vía el financiamiento: Alcances y limitaciones de las políticas de asignación. Espacio abierto. $16 \mathrm{~N}^{\circ}$ 1: 7-29. 2007.

GARCÍA GUADILLA, Carmen. Tensiones y transiciones. Educación superior latinoamericana en los albores del tercer milenio. Caracas, CENDES-UCV. 2004.

GARCÍA GUADILLA, Carmen. Informe de Venezuela. Proyecto CINDA: Educación superior en Iberoamérica 2006. Caracas, 2006. disponible en:

<www.carmengarciaguadilla.com/articulos/2006_EducSup_en_Iberoamerica.pdf> consultado el: 19 de agosto de 2015.

PARRA SANDOVAL, María Cristina. La educación superior en Iberoamérica 2011. La educación superior en Venezuela 2005-2009. Maracaibo, 2011. Disponible en: <http://www.cinda.cl/wp-content/uploads/2014/07/2011-Informe-Venezuela.pdf > Consulta el: 25 de julio de 2015.

PARRA SANDOVAL, María Cristina. Venezuela la política de educación superior desde el socialismo del siglo XXI. En Brunner y Villalobos. Políticas de educación superior en Iberoamérica, 2009-2013. Santiago de Chile, Ediciones Universidad Diego Portales. 2014.

SALMI, J. Y HAUPTMANN, A. Mecanismos de asignación de recursos en la educación superior: Tipología y evaluación", en Global University Network for Innovation. Educación superior en el mundo 2006. La Financiación de las universidades. Madrid, Ediciones Mundi-Prensa. 2006.

SANYAL B, C. Y MATIN, M. (2006) "La financiación de la educación superior: Perspectivas internacionales", en Global University Network for Innovation. Educación superior en el mundo 2006. La Financiación de las universidades. Madrid, Ediciones Mundi-Prensa. 2006.

REPÚBLICA BOLIVARIANA DE VENEZUELA. Reglamento $\mathbf{N}^{\circ} \mathbf{1}$ de la Ley Orgánica de la Administración Financiera del Sector Público, sobre el sistema presupuestario. Caracas, 2005. 